\title{
SOLUTION OF A PROBLEM OF BARENDREGT ON SENSIBLE $\lambda$-THEORIES
}

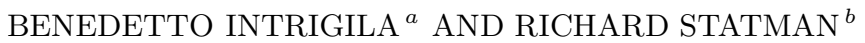 \\ ${ }^{a}$ Università degli Studi di Roma "Tor Vergata", Rome, Italy \\ e-mail address: intrigil@mat.uniroma2.it \\ ${ }^{b}$ Carnegie-Mellon University, Pittsburgh, PA, USA \\ e-mail address: rs31@andrew.cmu.edu
}

\begin{abstract}
H}$ is the theory extending $\beta$-conversion by identifying all closed unsolvables. $\mathcal{H} \omega$ is the closure of this theory under the $\omega$-rule (and $\beta$-conversion). A long-standing conjecture of $H$. Barendregt states that the provable equations of $\mathcal{H} \omega$ form a $\Pi_{1}^{1}$-complete set. Here we prove that conjecture.
\end{abstract}

\section{INTRODUCTION}

There is a strong need to make theorem provers such as COQ or ISABELL/HOL more and more powerful (see e.g. [3, 2, [1]). In particular it seems very hard to automatically set up inductive arguments to get universal conclusions. In this sense, the use of some (constructive) kind of $\omega$-rule is very appealing since one could get a universal conclusion from, say, a finite number of cases. Typically, this happens when for every property $P$ of interest, there exists a computable upper bound $k$ such that if every ground term of complexity less than $k$ satisfies $P$ then $\forall x . P(x)$ holds, so that a universal conclusion can be obtained e.g. by a systematic search on a finite set of cases.

Therefore, it is important to precisely assess the logical power of the $\omega$-rule in the different computational contexts. Here we consider the $\omega$-rule in the $\lambda \beta$-calculus. We have already considered constructive forms of such rule in [6], obtaining recursively enumerable $\lambda$-theories which are closed under the $\omega$-rule. Moreover, in [7, we have considered the more important problem of the $\omega$-rule added to the pure $\lambda \beta$-calculus. We have shown that the resulting theory is not recursively enumerable, by giving a many-one reduction of the set of true $\Pi_{2}^{0}$ sentences to the set of consequences of the lambda calculus with the $\omega$-rule. This solved in the affirmative a well known problem of $\mathrm{H}$. Barendregt 4, 5. More recently we have obtained the result ([8]) that such theory is not even arithmetical.

Here we consider the problem of determining the computational power of the $\omega$-rule added to the theory $\mathcal{H}$. $\mathcal{H}$ is the theory obtained extending $\lambda \beta$ by identifying all closed unsolvable terms. We prove that the resulting theory $\mathcal{H} \omega$ is $\boldsymbol{\Pi}_{1}^{1}$-complete, which solves another long-standing conjecture of H. Barendregt (see 4] Conjecture 17.4.15).

2000 ACM Subject Classification: F.4.1.

Key words and phrases: lambda calculus; sensible theories; omega rule. 


\section{The System $\mathcal{H} \omega$}

Notation will be standard and we refer to [4, for terminology and results on $\lambda$-calculus. In particular:

- $\equiv$ denotes syntactical identity;

- by the notation $[X / x] Y$ we mean the replacement of term $X$ for the variable $x$ inside $Y$, with the usual proviso that no free variable $y$ of $X$, with $y \not \equiv x$, becomes bounded after the substitution (see [4] 2.1.11-2.1.14);

- $\longrightarrow_{\beta}, \longrightarrow_{\eta}$ and $\longrightarrow_{\beta \eta}$ denote $\beta$-, $\eta$ - and, respectively, $\beta \eta$-reduction and $\longrightarrow_{\beta}^{*} \longrightarrow_{\eta}^{*}$ and $\longrightarrow{ }_{\beta \eta}^{*}$ their respective reflexive and transitive closures;

- $=\beta$ and $=\beta \eta$ denote $\beta$ - and, respectively, $\beta \eta$-conversion;

- combinators (i.e. closed $\lambda$-terms) such e.g. I have the usual meaning;

- $\underline{k}$ denotes the $k$-th Church numeral.

$\lambda$-terms are denoted by capital letters: in particular we adopt the convention that $M, N, P$, $Q, \ldots$ are closed terms and $U, V, X, Y, W, Z$ are possibly open terms.

The notion of $\lambda$-theory has the usual meaning of [4] Ch.4, that is a consistent set of equations between closed terms, which is closed under the axioms and the rules of $\lambda \beta$ calculus. We now briefly recall the $\lambda$-theories we are concerned with.

By $\lambda \beta$ we denote pure $\beta$-convertibility (see [4]). $\mathcal{H}$ is the $\lambda$-theory extending $\lambda \beta$ by identifying all closed unsolvable terms, see [4 Definition 4.1.6. We recall that this $\lambda$-theory can be formulated by adding to $\lambda \beta$ all equations of the form $M=\Omega$, where $M$ is a closed unsolvable term, the combinator $\Omega$ is defined as $\omega \omega$ and $\omega$ is $\lambda x . x x$. Moreover, we recall also that $\mathcal{H}$ is generated by the notion of reduction $\beta \boldsymbol{\Omega}$, see [4] Lemma 16.1.2. The notion of reduction $\beta \Omega$ is defined by adding to the $\beta$-reduction rule, the (non constructive) reduction rule:

$$
M \longrightarrow \boldsymbol{\Omega} \text { if } M \text { is unsolvable and } M \not \equiv \boldsymbol{\Omega}
$$

see 4] Section 15.2.

$\mathcal{H} \omega$ is the $\lambda$-theory obtained by adding the so called $\omega$-rule to $\mathcal{H}$, see [4] Definition 4.1.10 and Paragraph 4.2.

We formulate $\mathcal{H} \omega$ differently. As the reader will see, we want a formulation of the theory such that only equalities between closed terms can be proven.

Definition 2.1. Equality in $\mathcal{H} \omega$ (denoted by $={ }_{\omega}$ ) is defined by the following axioms and rules:

(1) Identity Axioms:

$$
M={ }_{\omega} M
$$

(2) Weak $\beta \boldsymbol{\Omega}$-Conversion Axioms:

$$
\begin{aligned}
(\lambda x . U) N & ={ }_{\omega}[N / x] U & & \text { (with }(\lambda x \cdot U) N \text { closed) } \\
{[N / x] U } & ={ }_{\omega}[N / x] U & & \text { (with }(\lambda x . U) N \text { closed) } \\
M & ={ }_{\omega} \Omega & & \text { (with } M \text { closed and unsolvable) } \\
\boldsymbol{\Omega} & ={ }_{\omega} M & & \text { (with } M \text { closed and unsolvable) }
\end{aligned}
$$

(3) Leibnitz Rule: Substitute Equals for Equals:

$$
\frac{[M / z] X={ }_{\omega}[M / z] Y \quad M={ }_{\omega} N}{[N / z] X={ }_{\omega}[N / z] Y}
$$


where terms $X$ and $Y$ have possibly $z$ as free variable, and no other free variable.

(4) The $\omega$-Rule:

$$
\frac{\forall M, M \text { closed, } P M={ }_{\omega} Q M}{P={ }_{\omega} Q}
$$

We call $\mathcal{H} \omega$ the $\lambda$-theory specified above. In the next Section we prove that this formulation gives rise to the same theory of $\underline{4}$.

\section{Derived Rules}

Now we prove that some rules are derived rules in $\mathcal{H} \omega$.

Proposition 3.1. (Symmetry) For every $M$ and $N$, if $M={ }_{\omega} N$ then $N={ }_{\omega} M$.

Proof. Axioms and rules of $\mathcal{H} \omega$ are completely symmetric, so a proof of $M={ }_{\omega} N$ can be converted into one of $N={ }_{\omega} M$ by reversing sides.

Proposition 3.2. (Transitivity) For every $M, N$ and $P$, if $M={ }_{\omega} N$ and $N={ }_{\omega} P$ then $M={ }_{\omega} P$.

Proof. Assume $M={ }_{\omega} N$. So, $N={ }_{\omega} M$. Then let $X$ be $z$ and $Y$ be $M$. We have that $[N / z] X={ }_{\omega}[N / z] Y$ and

$$
\frac{[N / z] X={ }_{\omega}[N / z] Y \quad N={ }_{\omega} P}{P={ }_{\omega} M}
$$

by one application of the Leibnitz Rule.

By $\beta \boldsymbol{\Omega}$-convertibility we mean the convertibility relation generated by the $\beta \boldsymbol{\Omega}$-reduction mentioned above (see 4] Paragraph 15.2). By a context $Z$ [ ] we mean a term with holes in the sense of [4] Definition 2.1.18.

Proposition 3.3. For closed $M$ and $N$, if $M \beta \boldsymbol{\Omega}$-converts to $N$ then $M={ }_{\omega} N$.

Proof.

(1) Let a context $Z[]$ and terms $\lambda x . U, V$ be given. Let $z_{1} \ldots z_{t}$ be an enumeration of all free variables occurring in $Z[], \lambda x . U$ and $V$.

By induction on the complexity of $Z[]$ one can prove that for all closed $P_{1} \ldots P_{t}$ we have:

$$
\left[P_{1} / z_{1}, \ldots, P_{t} / z_{t}\right] Z[(\lambda x . U) V]={ }_{\omega}\left[P_{1} / z_{1}, \ldots, P_{t} / z_{t}\right] Z[[V / x] U] .
$$

Thus for $\lambda$-closures $\lambda z_{1} \ldots z_{t} . Z[(\lambda x . U) V]$ and $\lambda z_{1} \ldots z_{t} . Z[[V / x] U]$, we have that for all closed $P_{1} \ldots P_{t}$ :

$$
\left(\lambda z_{1} \ldots z_{t} . Z[(\lambda x . U) V]\right) P_{1} \ldots P_{t}={ }_{\omega}\left(\lambda z_{1} \ldots z_{t} . Z[[V / x] U]\right) P_{1} \ldots P_{t}
$$

so $\lambda z_{1} \ldots z_{t} . Z[(\lambda x . U) V]={ }_{\omega} \lambda z_{1} \ldots z_{t} . Z[[V / x] U]$ by $t$ applications of the $\omega$-rule.

(2) Let a context $Z[]$ and an unsolvable term $V$ be given. Let $z_{1} \ldots z_{t}$ be an enumeration of all free variables occurring in $Z[]$ and $V$.

By induction on the complexity of $Z[]$ one can prove that for all closed $P_{1} \ldots P_{t}$ we have $\left[P_{1} / z_{1}, \ldots, P_{t} / z_{t}\right] Z[V]={ }_{\omega}\left[P_{1} / z_{1}, \ldots, P_{t} / z_{t}\right] Z[\Omega]$. Thus for $\lambda$-closures: $\lambda z_{1} \ldots z_{t} . Z[V]$ and $\lambda z_{1} \ldots z_{t} . Z[\Omega]$, we have that for all closed $P_{1} \ldots P_{t}$ :

$$
\left(\lambda z_{1} \ldots z_{t} . Z[V]\right) P_{1} \ldots P_{t}={ }_{\omega}\left(\lambda z_{1} \ldots z_{t} . Z[\Omega]\right) P_{1} \ldots P_{t}
$$

so $\lambda z_{1} \ldots z_{t} . Z[V]={ }_{\omega} \lambda z_{1} \ldots z_{t} . Z[\Omega]$ by $t$ applications of the $\omega$-rule. 
Proposition 3.4. If $X \beta \boldsymbol{\Omega}$-converts to $Y$ then the $\lambda$-closures of $X$ and $Y$ are provably equal in $\mathcal{H} \omega$.

Proof. The proposition follows directly from the previous one.

By the previous results it follows that $\mathcal{H} \omega$ is exactly the same theory defined in [4.

We observe also the following:

Proposition 3.5. The so-called $\eta$-conversion (that is $(\lambda x . M x)=M$ ) obviously holds in $\mathcal{H} \omega$, for any closed term $M$. (With respect to the usual formulation of the $\eta$-conversion, observe that since $M$ is closed there is no need to require $x$ fresh in $M$ ).

\section{WEAK $\beta \Omega$-REDUCTION}

We call weak $\beta \boldsymbol{\Omega}$-conversion the smallest congruence relation containing the equations of Weak $\beta \Omega$-Conversion Axioms above (see Definition 2.12). Observe that this relation also includes the Identity Axioms. We write $M \sim_{w \beta \Omega} N$ to denote the weak $\beta \boldsymbol{\Omega}$-conversion relation. Moreover such equations can be oriented, giving rise to the following contraction rules:

weak $\beta$-contraction rule

$$
(\lambda x . M) N \longrightarrow{ }_{w \beta}[N / x] M \quad(\text { with }(\lambda x . M) N \text { closed })
$$

weak $\boldsymbol{\Omega}$-contraction rule

$$
M \longrightarrow \Omega \quad \text { (with } M \text { closed and unsolvable and } M \not \equiv \boldsymbol{\Omega})
$$

We call weak $\beta \boldsymbol{\Omega}$-reduction the reduction relation generated by the two rules, after closure under contexts (see [4 3.1). It is easy to see that the weak $\beta \boldsymbol{\Omega}$-conversion is the convertibility relation generated by the weak $\beta \boldsymbol{\Omega}$-reduction. We shall call the two contraction rules above also weak $\beta$-reduction rule and, respectively weak $\boldsymbol{\Omega}$-reduction rule. This terminology includes the case that such rules are applied inside a context.

We write:

$$
\longrightarrow w \beta \Omega \text { and } \longrightarrow_{w \beta \Omega}^{*}
$$

to denote weak $\beta \boldsymbol{\Omega}$-reduction and, respectively, its reflexive and transitive closure.

We recall a result needed in the following.

Proposition 4.1. Weak $\beta \boldsymbol{\Omega}$-reduction is Church-Rosser.

Proof. Weak $\beta$ - and, respectively, weak $\boldsymbol{\Omega}$-reductions are both Church-Rosser and commute. Now, use the Hindley-Rosen Lemma (see [4, 3.3.5).

In the sequel, we shall need the following notions on reductions. We define the notions of trace and extended trace (etrace) as follows. Given the reduction $F \longrightarrow_{\beta}^{*} G$ (or the reduction $F \longrightarrow_{w \beta \Omega}^{*} G$ ) and the closed subterm $M$ of $F$, the traces of $M$ in the terms of the reduction are simply the copies of $M$ until each is either deleted by a contraction of a redex with a dummy lambda, replaced by $\boldsymbol{\Omega}$ by an $\boldsymbol{\Omega}$-reduction (possibly of a superterm $M^{\prime}$ of $M$ ) or altered by a reduction internal to $M$ or a reduction with $M$ at the head (when $M$ begins with lambda or when the reduction is an $\boldsymbol{\Omega}$-reduction). The notion of etrace is the same except that we allow internal reductions, so that a copy of $M$ altered by an internal reduction continues to be an etrace. 


\section{Normal Form for $\mathcal{H} \omega$ Proofs}

As usual proofs in $\mathcal{H} \omega$ can be thought of as (possibly infinite) well-founded trees. We distinguish between two cases.

- The proof ends with an application of the $\omega$-rule.

- Otherwise. So, we can consider all nodes of the proof tree that have no premises of the $\omega$-rule as descendant. Or, in other terms, there are no occurrences of the $\omega$-rule in the path from the node to the conclusion of the proof. We call the set of such nodes the endpiece of the proof.

Notice that the endpiece of a proof consists of a finite tree of Leibnitz Rule inferences all of whose leaves are either instances of the Identity Axioms, instances of the Weak $\beta \boldsymbol{\Omega}$ Conversion Axioms, or direct conclusions of the $\omega$-rule. The tree reduces to a single node in case the proof amounts to an instance of the Identity Axioms or to an instance of the Weak $\beta \boldsymbol{\Omega}$-Conversion Axioms. We shall put this endpiece into a normal form.

Definition 5.1. An endpiece is in normal form iff it is of the form:

$$
\begin{aligned}
& \frac{M={ }_{\omega} M \quad M \sim_{w \beta \Omega} M_{1} P_{1}}{M={ }_{\omega} M_{1} Q_{1}} P_{1}={ }_{\omega} Q_{1} \\
& \frac{M={ }_{\omega} M_{1} Q_{1}}{M_{1} Q_{1} \sim_{w \beta \Omega} M_{2} P_{2}}{ }_{P_{2}={ }_{\omega} M_{2} Q_{2}}
\end{aligned}
$$

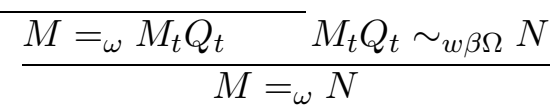

where each equality of the form $P_{i}={ }_{\omega} Q_{i}$, for $1 \leq i \leq t$, is a direct conclusion of the $\omega$-rule. We allow the degenerate case $t=0$ and consider in normal form:

- an instance of the Identity Axioms;

- as well as the endpiece:

$$
\frac{M={ }_{\omega} M \quad M \sim_{w \beta \Omega} N}{M={ }_{\omega} N}
$$

Remark. In the previous Definition, observe that the intuitive motivation of the notion of normal form is to have a mean to separate - into the endpiece of a proof - the conclusions of the $\omega$-rule from the other components of the endpiece itself. The normal form diagram represents a sequence of applications of the Leibnitz Rule, and in particular the odd lines are instances of the transitivity rule, while the even ones are substitutions of the term $Q_{i}$ for the equal term $P_{i}$ in the applicative context $M_{i}$ [ ]. Observe that the normal form diagram is not, strictly speaking, a proof tree in $\mathcal{H} \omega$, since premises of the form $M_{i} Q_{i} \sim_{w \beta \Omega} M_{i+1} P_{i+1}$ refer to the $\beta \boldsymbol{\Omega}$-conversion relation. (This explains why we need two degenerate cases, one when the whole proof is an instance of Identity Axioms, and the other one when we have a proof of the $\beta \boldsymbol{\Omega}$-convertibiliy of the terms $M$ and $N$. Of course, the former could also be considered a particular case of the latter). It is clear, however, by the result of Section 3 , that each $\beta \boldsymbol{\Omega}$-conversion can be expanded into a $\mathcal{H} \omega$ proof tree.

Theorem 5.2. For every proof $\mathcal{T}$ in $\mathcal{H} \omega$ there exists a proof $\mathcal{T}^{\prime}$ of the same conclusion with the endpiece in normal form.

We shall use several lemmata. 
Lemma 5.3. Proofs in normal forms are closed under symmetry and transitivity rules.

Proof.

- (symmetry)

To see this, observe that a proof in normal form of $M={ }_{\omega} N$ can be reversed into a proof in normal form of $N={ }_{\omega} M$. Indeed, each direct conclusion of the $\omega$-rule $P_{i}={ }_{\omega} Q_{i}$ can be reversed in a direct $\omega$-rule conclusion of $Q_{i}={ }_{\omega} P_{i}$, since for every premise $P_{i} R={ }_{\omega} Q_{i} R$ there is (by Proposition 3.1) a premise $Q_{i} R={ }_{\omega} P_{i} R$. Moreover the sequences $Q_{t}, Q_{t-1} \ldots Q_{1}$ and $P_{t}, P_{t-1} \ldots P_{1}$ take the place of $P_{1}, P_{2} \ldots P_{t}$ and, respectively, of $Q_{1}, Q_{2} \ldots Q_{t}$.

- (transitivity)

Given proofs in normal form of $M={ }_{\omega} N$ and of $N={ }_{\omega} P$, to obtain a proof in normal form of $M={ }_{\omega} P$ contract the last row of $M={ }_{\omega} N$ :

$$
\frac{M={ }_{\omega} M_{t} Q_{t} \quad M_{t} Q_{t} \sim_{w \beta \Omega} N}{M={ }_{\omega} N}
$$

and the first row of $N={ }_{\omega} P$ :

$$
\frac{N={ }_{\omega} N \quad N \sim_{w \beta \Omega} M_{1}^{\prime} Q_{1}^{\prime}}{N={ }_{\omega} M_{1}^{\prime} Q_{1}^{\prime}}
$$

as follows:

$$
\frac{M={ }_{\omega} M_{t} Q_{t} \quad M_{t} Q_{t} \sim_{w \beta \Omega} M_{1}^{\prime} Q_{1}^{\prime}}{M={ }_{\omega} M_{1}^{\prime} Q_{1}^{\prime}}
$$

and then follows the proof in normal form of $N={ }_{\omega} P$ replacing every left-side occurrence of $N$ with $M$.

Lemma 5.4. If there is a proof in normal form of $M={ }_{\omega} N$ then for every $X$, with a unique free variable $z$, there is a proof in normal form, with the same length, of $[M / z] X={ }_{\omega}[N / z] X$.

Proof. Let the proof of $M={ }_{\omega} N$ be of the form:

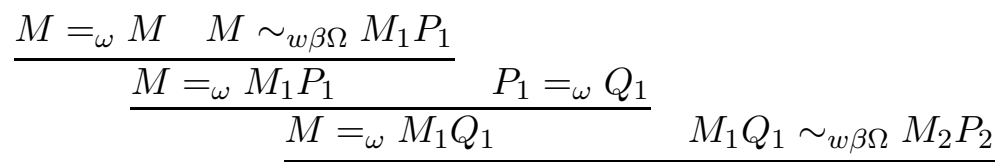

$$
\begin{aligned}
& \frac{M={ }_{\omega} M_{2} P_{2}}{\ldots}
\end{aligned}
$$

To get a proof in normal form of $[M / z] X={ }_{\omega}[N / z] X$, transform it as follows:

$$
\frac{\frac{[M / z] X={ }_{\omega}[M / z] X \quad[M / z] X \sim_{w \beta \Omega}\left(\lambda u .(\lambda z . X)\left(M_{1} u\right)\right) P_{1}}{[M / z] X=_{\omega}\left(\lambda u .(\lambda z \cdot X)\left(M_{1} u\right)\right) P_{1} \quad P_{1}={ }_{\omega} Q_{1}}}{\frac{[M / z] X={ }_{\omega}\left(\lambda u .(\lambda z \cdot X)\left(M_{1} u\right)\right) Q_{1}\left(\lambda u \cdot(\lambda z \cdot X)\left(M_{1} u\right)\right) Q_{1} \sim_{w \beta \Omega}\left(\lambda u .(\lambda z . X)\left(M_{2} u\right)\right) P_{2}}{\frac{[M / z] X=_{\omega}\left(\lambda u .(\lambda z \cdot X)\left(M_{2} u\right)\right) P_{2}}{\ldots}}}
$$


Lemma 5.5. Proofs with the endpiece in normal form are closed under Axioms and Rules of $\mathcal{H} \omega$.

Proof. We argue by induction on the complexity of the proof of $M={ }_{\omega} N$.

(1) If the proof consists of an instance of the identity axiom, then there is nothing to prove.

(2) If the proof consists of an instance of the Weak $\beta \boldsymbol{\Omega}$-Conversion Axioms, then use the fact that the sequences $P_{1}, \ldots, P_{t}$ and $Q_{1}, \ldots, Q_{t}$ can be empty (i.e. $t=0$ ).

(3) If the proof ends with an instance of the $\omega$-rule then it can be put in normal form as follows:

$$
\frac{M={ }_{\omega} M \quad M \sim_{w \beta \Omega} \mathbf{I} M}{\frac{M={ }_{\omega} \mathbf{I} M \quad M={ }_{\omega} N}{M={ }_{\omega} \mathbf{I} N} \quad \mathbf{I} N \sim_{w \beta \Omega} N}
$$

(4) Assume that the proof ends with an instance of the Leibnitz Rule of the form:

$$
\frac{[P / z] X={ }_{\omega}[P / z] Y \quad P={ }_{\omega} Q}{[Q / z] X={ }_{\omega}[Q / z] Y}
$$

with $M \equiv[Q / z] X$ and $N \equiv[Q / z] Y$. By induction hypothesis and Lemmata $[5.3$ and 5.4 there are proofs in normal form of:

$$
[P / z] X={ }_{\omega}[P / z] Y \quad, \quad[Q / z] X={ }_{\omega}[P / z] X \quad \text { and } \quad[P / z] Y={ }_{\omega}[Q / z] Y
$$

and therefore by Lemma 5.3 again, we get a proof in normal form of $[Q / z] X={ }_{\omega}[Q / z] Y$.

Remark. Observe that the transformation of an instance of the $\omega$-rule into a proof with an endpiece in normal form has only an auxiliary character. In other words, this transformation can be done if needed, but we do not want to perform it systematically.

Proof of Theorem 5.2. It is clear that, from the previous lemmas, Theorem 5.2 follows.

Remark. Theorem 5.2 is essentially a particular case of a general result about Leibnitz Rule due to the second author of the present paper. For more details the reader should consult [1].

Now consider a proof with an endpiece in normal form:

$$
\begin{aligned}
& M={ }_{\omega} M \quad M \sim_{w \beta \Omega} M_{1} P_{1}
\end{aligned}
$$

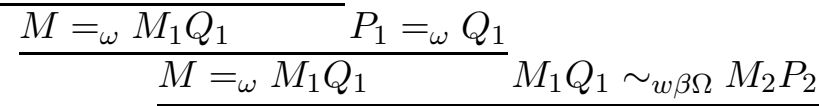

$$
\begin{aligned}
& \underline{M=}{ }_{\omega} M_{2} Q_{2} \quad P_{2}={ }_{\omega} Q_{2} \\
& \cdots
\end{aligned}
$$

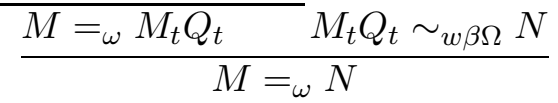

We represent this proof as a computation viz

$$
M \sim_{w \beta \Omega} M_{1} P_{1}={ }_{\omega} M_{1} Q_{1} \sim_{w \beta \Omega} M_{2} P_{2} \ldots \sim_{w \beta \Omega} M_{t} P_{t}={ }_{\omega} M_{t} Q_{t} \sim_{w \beta \Omega} N
$$

Remark. We include the degenerate cases as follows:

- $M={ }_{\omega} M$ gives rise to the computation $M \sim_{w \beta \Omega} M$;

- $M \sim_{w \beta \Omega} N$ can be directly considered as a computation. 
Definition 5.6. We shall call the sequence (5.1) the end piece computation of a proof.

\section{ORdinals}

Since proofs are infinite trees $\mathcal{T}$ they can be described by countable ordinals. In the following, we shall need a few facts about countable ordinals. For completeness, we recall the main notions involved. For more details, see e.g. [10.

(a) Cantor Normal Form to the Base Omega $(\omega)$. Every countable ordinal $\alpha$ can be written uniquely in the form $\omega^{\alpha_{1}} * n_{1}+\ldots+\omega^{\alpha_{k}} * n_{k}$ where $n_{1}, \ldots, n_{k}$ are positive integers and $\alpha_{1}>\ldots>\alpha_{k}$ are ordinals.

Note that in the special case when $\alpha$ is a fixed point of ordinal exponentiation (like $\epsilon_{0}$ ) we have $\omega^{\alpha} * 1$ as Cantor normal form of $\alpha$.

(b) Hessenberg Sum. Write $\alpha=\omega^{\alpha_{1}} * n_{1}+\ldots+\omega^{\alpha_{k}} * n_{k}$ and $\gamma=\omega^{\alpha_{1}} * m_{1}+\ldots+\omega^{\alpha_{k}} * m_{k}$ where some of the $n_{i}$ and $m_{j}$ may be 0 . Then the Hessenberg Sum is defined as follows: $\alpha \oplus \gamma={ }_{\text {def }} \omega^{\alpha_{1}} *\left(n_{1}+m_{1}\right)+\ldots+\omega^{\alpha_{k}} *\left(n_{k}+m_{k}\right)$.

The Hessenberg sum is strictly increasing on both arguments. That is, for $\alpha, \gamma$ different from 0, we have: $\alpha, \gamma<\alpha \oplus \gamma$.

(c) Hessenberg Product . We only need this for product with an integer. We put: $\alpha \odot n={ }_{\text {def }} \alpha \oplus \ldots \oplus \alpha$-times.

Coming back to proofs, observe first that we can assume that if a proof has an endpiece, then this endpiece is in normal form (see the previous Section). The ordinal that we want to assign to a proof $\mathcal{T}$ (considered as a tree) is the transfinite ordinal ord $(\mathcal{T})$, the order of $\mathcal{T}$, defined recursively by:

Definition 6.1. Let $\oplus$ be the Hessenberg sum of ordinals defined above.

- If $\mathcal{T}$ ends in an endpiece computation of the form (5.1) and we are in the degenerate case $t=0$ then $\operatorname{ord}(\mathcal{T})={ }_{\text {def }} 1$;

- If $\mathcal{T}$ ends in an instance of the $\omega$-rule whose premisses have trees resp. $\mathcal{T}_{1}, \ldots \mathcal{T}_{i}, \ldots$ then $\operatorname{ord}(\mathcal{T})={ }_{\text {def }} \omega^{\theta}$, with $\theta=\operatorname{Sup}\left\{\operatorname{ord}\left(\mathcal{T}_{1}\right) \oplus \ldots \oplus \operatorname{ord}\left(\mathcal{T}_{i}\right): i=1,2, \ldots\right\} ;$

- If $\mathcal{T}$ ends in an endpiece computation of the form (5.1), with $t>0$, and the equations $P_{1}={ }_{\omega} Q_{1}, \ldots, P_{t}={ }_{\omega} Q_{t}$, have resp. trees $\mathcal{T}_{1}, \ldots, \mathcal{T}_{t}$ then $\operatorname{ord}(\mathcal{T})={ }_{\text {def }} 1 \oplus \operatorname{ord}\left(\mathcal{T}_{1}\right) \oplus \ldots \oplus$ $\operatorname{ord}\left(\mathcal{T}_{t}\right)$.

Fact 1. If $\mathcal{T}$ ends in an endpiece computation of the form (5.1), with $t>0$, and the equations $P_{1}={ }_{\omega} Q_{1}, \ldots, P_{t}={ }_{\omega} Q_{t}$, have resp. trees $\mathcal{T}_{1}, \ldots, \mathcal{T}_{t}$ then $\operatorname{ord}(\mathcal{T})>\operatorname{ord}\left(\mathcal{T}_{i}\right)$, for each $i=1, \ldots, t$.

Proof. $\operatorname{ord}\left(\mathcal{T}_{i}\right)>0$ and $\oplus$ is strictly increasing on its arguments.

Fact 2. Assume that $\mathcal{T}$ ends in an instance of the $\omega$-rule whose premisses have, respectively, trees $\mathcal{T}_{1}, \ldots, \mathcal{T}_{t}, \ldots$ Then for any integers $t, n_{1}, \ldots, n_{t}$

$$
\operatorname{ord}(\mathcal{T})>\operatorname{ord}\left(\mathcal{T}_{1}\right) \odot n_{1} \oplus \ldots \oplus \operatorname{ord}\left(\mathcal{T}_{t}\right) \odot n_{t} .
$$

Proof. Let $\operatorname{ord}\left(\mathcal{T}_{i}\right)=\alpha_{i}$, for $1 \leq i \leq t$ and put all $\alpha_{1}, \ldots, \alpha_{t}$ into Cantor normal form:

$$
\alpha_{1}=\omega^{\beta_{1}} * n_{11}+\ldots+\omega^{\beta_{k}} * n_{1 k} \quad \ldots \quad \alpha_{t}=\omega^{\beta_{1}} * n_{t 1}+\ldots+\omega^{\beta_{k}} * n_{t k} .
$$


Let $n=\max \left\{n_{r}, n_{j 1}\right\}+1$, with $j, r=1 \ldots t$. Then

$$
\left.\alpha_{1} \odot n_{1} \oplus \ldots \oplus \alpha_{t} \odot n_{t}<\alpha_{1} \odot n \oplus \ldots \oplus \alpha_{t} \odot n=\alpha_{1} \oplus \ldots \oplus \alpha_{t}\right) \odot n \leq \omega^{\beta_{1}} * n^{2} * t .
$$

Now let $\theta=\operatorname{Sup}\left\{\operatorname{ord}\left(\mathcal{T}_{1}\right) \oplus \ldots \oplus \operatorname{ord}\left(\mathcal{T}_{i}\right): i=1,2, \ldots\right\}$. We have $\omega^{\beta_{1}}<\theta \leq \omega^{\theta}=\operatorname{ord}(\mathcal{T})$. But $\operatorname{ord}(\mathcal{T})$ is a countable ordinal of the form $\omega^{\gamma}$ and is thus closed under addition. Hence $\omega^{\beta_{1}} * n^{2} * t<\operatorname{ord}(\mathcal{T})$.

\section{Cascades of Beta Reductions}

Recall that, as usual, we consider only closed terms. We define the set of weak $\beta \boldsymbol{\Omega}$ head normal forms (whnf) as follows:

(1) an unsolvable term is in whnf iff it is $\boldsymbol{\Omega}$;

(2) a solvable term is in whnf iff it has not a head weak $\beta$-redex that is it has not the form $\lambda x_{1} \ldots x_{n} .(\lambda x . U) V M_{1} \cdots M_{k}$, with $(\lambda x . U)$ and $V$ closed.

Now we want to prove that the set weak $\beta \boldsymbol{\Omega}$ head normal forms is cofinal w.r.t. weak $\beta \boldsymbol{\Omega}$-reduction, in the sense of the following theorem.

Theorem 7.1. For every $M$ there exists an $N$ in whnf, such that $M \longrightarrow{ }_{w \beta \Omega}^{*} N$

Proof. If $M$ is unsolvable, then $M \longrightarrow_{w \beta \Omega}^{*} \Omega$.

Assume $M$ solvable. Then $M \longrightarrow_{\beta}^{*} M^{\prime}$ by a sequence of head $\beta$-reductions, where $M^{\prime}$ has the form $\lambda x_{1} \ldots x_{n} . x_{i} V_{1} \ldots V_{m}$ (see [4 8.3.11). If every $\beta$-reduction is a weak one then take $N \equiv M^{\prime}$, otherwise $N$ is the first term in the sequence where a weak head $\beta$-reduction cannot be performed.

By the Church-Rosser theorem for weak $\beta \boldsymbol{\Omega}$-reductions, an endpiece computation

$$
M \sim_{w \beta \Omega} M_{1} P_{1}={ }_{\omega} M_{1} Q_{1} \sim_{w \beta \Omega} M_{2} P_{2} \ldots \sim_{w \beta \Omega} M_{t} P_{t}={ }_{\omega} M_{t} Q_{t} \sim_{w \beta \Omega} N
$$

can be put in the form (that we still call an endpiece computation)

$$
\begin{gathered}
M \longrightarrow{ }_{w \beta \Omega}^{*} R_{1}{ }_{w \beta \Omega}^{*} \longleftarrow M_{1} P_{1}={ }_{\omega} M_{1} Q_{1} \longrightarrow_{w \beta \Omega}^{*} R_{2}{ }_{w \beta \Omega}^{*} \longleftarrow M_{2} P_{2} \\
\ldots \longrightarrow{ }_{w \beta \Omega}^{*} M_{t} P_{t}={ }_{w} M_{t} Q_{t} \longrightarrow{ }_{w \beta \Omega}^{*} R_{t+1}{ }_{w \beta \Omega}^{*} \longleftarrow N
\end{gathered}
$$

Now, we want to show that special conditions can be imposed on the weak $\beta \boldsymbol{\Omega}$-reductions occurring in each endpiece computation.

Definition 7.2. An endpiece computation of the form

$$
\begin{gathered}
M \longrightarrow{ }_{w \beta \Omega}^{*} R_{1}{ }_{w \beta \Omega}^{*} \longleftarrow M_{1} P_{1}={ }_{\omega} M_{1} Q_{1} \longrightarrow{ }_{w \beta \Omega}^{*} R_{2}{ }_{w \beta \Omega}^{*} \longleftarrow M_{2} P_{2} \\
\ldots \longrightarrow{ }_{w \beta \Omega}^{*} M_{t} P_{t}={ }_{\omega} M_{t} Q_{t} \longrightarrow{ }_{w \beta \Omega}^{*} R_{t+1}{ }_{w \beta \Omega}^{*} \longleftarrow N
\end{gathered}
$$

is called a a cascade of weak $\beta \boldsymbol{\Omega}$-reductions iff

(1) all the confluence terms $R_{i}, 1 \leq i \leq t+1$ are in whnf;

(2) all the reductions of the form $R_{i} \underset{w \beta \Omega}{*} M_{i} P_{i}$, with $1 \leq i \leq t$ occurring in the endpiece are one step $\beta$-reductions of the form $\left[P_{i} / x\right] X \quad w \beta \Omega \longleftarrow(\lambda x . X) P_{i}$, for some $X$, and moreover such $X$ has not the form $\lambda y_{1} \ldots y_{r} . x X_{1} \cdots X_{m}$.

Note that this puts no restriction on left facing arrows.

Definition 7.3. The notion of a cascaded proof is defined inductively as follows. 
(1) A proof with a degenerate endpiece is a cascaded proof if it has the form:

$$
M \longrightarrow \underset{w \beta \Omega}{*} R \underset{w \beta \Omega}{*} \longleftarrow N
$$

with $R$ in whnf.

(2) A proof ending with an instance of the $\omega$-rule is a cascaded proof if the proofs of the premisses of the instance are cascaded.

(3) Otherwise a proof is cascaded if its endpiece is a cascade of weak $\beta \boldsymbol{\Omega}$-reductions and all the proofs of the leaves which are direct conclusions of the $\omega$-rule are cascaded.

In the following, we need the following well known fact about $\mathcal{H} \omega$.

Proposition 7.4. If $M={ }_{\omega} N$ then $\mathrm{BT}(M)={ }_{\eta} \mathrm{BT}(N)$, that is $M$ and $N$ have $\eta$-equal Böhm trees.

Proof. By Proposition 16.2.7 of [4], this holds for equality in the theory $\mathcal{H}^{*}$. Moreover, by Section 17.2 of [4, we have that $\mathcal{H} \omega$ is included in $\mathcal{H}^{*}$.

Now, we want to prove the following important fact about cascaded proofs.

Proposition 7.5. If $M={ }_{\omega} N$ then there is a cascaded proof of $M={ }_{\omega} N$.

Proof. We prove this proposition by induction on the ordinal $\operatorname{ord}(\mathcal{T})$ of a proof $\mathcal{T}$ in normal form of $M={ }_{\omega} N$.

For the base case just suppose that $M \sim_{w \beta \Omega} N$ and use the Church-Rosser theorem.

Induction step. Assume first that $M={ }_{\omega} N$ is the direct conclusion of the $\omega$-rule. This follows directly from the induction hypothesis.

Otherwise, $M={ }_{\omega} N$ is the conclusion of a chain of equality inferences:

$$
M \sim_{w \beta \Omega} M_{1} P_{1}={ }_{\omega} M_{1} Q_{1} \sim_{w \beta \Omega} M_{2} P_{2} \sim_{w \beta \Omega} \ldots \sim_{w \beta \Omega} M_{t} P_{t}={ }_{\omega} M_{t} Q_{t} \sim_{w \beta \Omega} N
$$

where $t>0$ and each $M_{i} P_{i}={ }_{\omega} M_{i} Q_{i}$ is the conclusion of an instance of the $\omega$-rule. Again by the Church-Rosser theorem we have the following computation:

$$
\begin{gathered}
M \longrightarrow{ }_{w \beta \Omega}^{*} R_{1}{ }_{w \beta \Omega}^{*} \longleftarrow M_{1} P_{1}={ }_{\omega} M_{1} Q_{1} \longrightarrow_{w \beta \Omega}^{*} R_{2}{ }_{w \beta \Omega}^{*} \longleftarrow M_{2} P_{2} \longrightarrow_{w \beta \Omega}^{*} \\
\ldots \longrightarrow{ }_{w \beta \Omega}^{*} R_{t+1} \stackrel{*}{*}{ }_{w \beta \Omega} \longleftarrow N .
\end{gathered}
$$

Clearly each $R_{i}$ can be replaced by any weak $\beta \Omega$-reduct of $R_{i}$.

Consider a reduction from $M_{1} P_{1}$ to $R_{1}$ with all the weak $\Omega$-reductions (that is reductions of the form $\longrightarrow \Omega$ ) at the end; such a reduction exists by [4 Proposition 15.2.9. Moreover, we can assume that no term in the reduction is unsolvable, for otherwise $M$ and $N$ are both unsolvable, by the previous proposition, and we simply have the cascaded proof $M \longrightarrow w \beta \Omega \boldsymbol{\Omega}_{w \beta \Omega} \longleftarrow N$.

We follow all etraces of $P_{1}$ in the reduction of $M_{1} P_{1}$ to $R_{1}$ attempting to simulate this with a reduction of $M_{1} Q_{1}$. On the $M_{1} Q_{1}$ side we skip reductions internal to etraces of $P_{1}$. When we come to redexes $(\lambda u . U) V$ where $P_{1} \longrightarrow{ }_{w \beta \Omega}^{*} \lambda u . U$, let $V=\left[V_{1} / x_{1}, \ldots, V_{r} / x_{r}\right] X$ showing all the etraces of $P_{1}$ in $V$. Then:

$$
Q_{1}\left(\left[Q_{1} / x_{1}, \ldots, Q_{1} / x_{r}\right] X\right)={ }_{\omega}\left[\left(\left[Q_{1} / x_{1}, \ldots, Q_{1} / x_{r}\right] X\right) / u\right] U
$$

(via the equality $Q_{1}\left(\left[Q_{1} / x_{1}, \ldots, Q_{1} / x_{r}\right] X\right)={ }_{\omega} P_{1}\left(\left[Q_{1} / x_{1}, \ldots, Q_{1} / x_{r}\right] X\right)$ ) by a proof with ordinal (much) less than $\operatorname{ord}(\mathcal{T})$. So, in the $M_{1} Q_{1}$ side we replace the reduction of $(\lambda u . U) V$, taking place in the $M_{1} P_{1}$ side, with the computation:

$$
Q_{1}\left(\left[Q_{1} / x_{1}, \ldots, Q_{1} / x_{r}\right] X\right)={ }_{\omega} P_{1}\left(\left[Q_{1} / x_{1}, \ldots, Q_{1} / x_{r}\right] X\right) \longrightarrow w \beta \Omega\left[\left[Q_{1} / x_{1}, \ldots, Q_{1} / x_{r}\right] X / u\right] U .
$$


Assume now that, in the $M_{1} P_{1}$ side, we come to an $\Omega$-reduction containing etraces of $P_{1}$, say of the form $U \longrightarrow{ }_{w \beta \Omega} \Omega$. Then if we replace every occurrence of etraces of $P_{1}$ in $U$ with $Q_{1}$, we obtain - by the previous proposition - a term $U^{\prime}$ which is also unsolvable. So, in the $M_{1} Q_{1}$ side, we perform the reduction $U^{\prime} \longrightarrow{ }_{w \beta \Omega} \Omega$.

In the end we obtain $R_{1}$ as $\left[V_{1} / x_{1}, \ldots, V_{r} / x_{r}\right] X$ for some $X$ where $V_{1}, \ldots, V_{r}$ are the remaining etraces of $P_{1}$. On the $M_{1} Q_{1}$ side we obtain $\left[Q_{1} / x_{1}, \ldots, Q_{1} / x_{r}\right] X$. Since there are only finitely many instances of $(*)$, we have that $\left[Q_{1} / x_{1}, \ldots, Q_{1} / x_{r}\right] X={ }_{\omega} N$ by a proof with ordinal $<\operatorname{ord}(\mathcal{T})$ (use Fact 2 of the Section [6). Thus there exists a cascaded proof $\mathcal{T}^{+}$of $\left[Q_{1} / x_{1}, \ldots, Q_{1} / x_{r}\right] X={ }_{\omega} N$.

Subcase 1. $X \equiv x$ so that $R_{1}$ is an etrace of $P_{1}$.

Let $L$ be given. Since there are only finitely many instances of $\left(^{*}\right)$, we have that $M L={ }_{\omega} N L$ by a proof with ordinal $<\operatorname{ord}(\mathcal{T})$ (again, use Fact 2 of the Section 6 ). Thus there exists a cascaded proof of $M L={ }_{\omega} N L$. Since this holds for every $L$ we obtain a cascaded proof $M={ }_{\omega} N$, by an application of the $\omega$-rule with cascaded proofs for all the premisses.

Subcase 2. Otherwise.

By the Church-Rosser theorem there exists a common reduct $V$ of all the $V_{1}, \ldots, V_{r}$. In addition, by induction hypothesis, there exists a cascaded proof $\mathcal{T}^{++}$of $V={ }_{\omega} Q_{1}$. We distinguish two cases.

Subcase 2.1. $X$ begins with some variable $x_{j}$, say $X \equiv x_{j} X_{1} \ldots X_{s}$.

In this case, since there are only finitely many instances of $\left(^{*}\right)$, to which we add a proof of:

$$
\begin{aligned}
& V_{j}\left(\left[Q_{1} / x_{1}, \ldots, Q_{1} / x_{r}\right] X_{1}\right) \ldots\left(\left[Q_{1} / x_{1}, \ldots, Q_{1} / x_{r}\right] X_{s}\right)={ }_{\omega} \\
& ={ }_{\omega} Q_{1}\left(\left[Q_{1} / x_{1}, \ldots, Q_{1} / x_{r}\right] X_{1}\right) \ldots\left(\left[Q_{1} / x_{1}, \ldots, Q_{1} / x_{r}\right] X_{s}\right)
\end{aligned}
$$

we have that $V_{j}\left(\left[Q_{1} / x_{1}, \ldots, Q_{1} / x_{r}\right] X_{1}\right) \ldots\left(\left[Q_{1} / x_{1}, \ldots, Q_{1} / x_{r}\right] X_{s}\right)={ }_{\omega} N$ has a proof with ordinal $<\operatorname{ord}(\mathcal{T})$. So, there exists a cascaded proof $\mathcal{T}^{*}$ of this equality.

Thus, in this case, the desired cascaded proof of $M={ }_{\omega} N$ is obtained concatenating the following pieces:

(1) $M \longrightarrow{ }_{w \beta \Omega}^{*} V_{j}\left(\left[V / x_{1}, \ldots, V / x_{r}\right] X_{1}\right) \ldots\left(\left[V / x_{1}, \ldots, V / x_{r}\right] X_{s}\right)$

(2) $V_{j}\left(\left[V / x_{1}, \ldots, V / x_{r}\right] X_{1}\right) \ldots\left(\left[V / x_{1}, \ldots, V / x_{r}\right] X_{s}\right) \quad w \beta \Omega \longleftarrow$

$w \beta \Omega \longleftarrow\left(\lambda x . V_{j}\left(\left[x / x_{1}, \ldots, x / x_{r}\right] X_{1}\right) \ldots\left(\left[x / x_{1}, \ldots, x / x_{r}\right] X_{s}\right)\right) V$

(3) $\left(\lambda x . V_{j}\left(\left[x / x_{1}, \ldots, x / x_{r}\right] X_{1}\right) \ldots\left(\left[x / x_{1}, \ldots, x / x_{r}\right] X_{s}\right)\right) V={ }_{\omega}$

$\left(\lambda x . V_{j}\left(\left[x / x_{1}, \ldots, x / x_{r}\right] X_{1}\right) \ldots\left(\left[x / x_{1}, \ldots, x / x_{r}\right] X_{s}\right)\right) Q_{1}$

(4) $\left(\lambda x . V_{j}\left(\left[x / x_{1}, \ldots, x / x_{r}\right] X_{1}\right) \ldots\left(\left[x / x_{1}, \ldots, x / x_{r}\right] X_{s}\right)\right) Q_{1} \longrightarrow w \beta \Omega$

$\longrightarrow w \beta \Omega V_{j}\left(\left[Q_{1} / x_{1}, \ldots, Q_{1} / x_{r}\right] X_{1}\right) \ldots\left(\left[Q_{1} / x_{1}, \ldots, Q_{1} / x_{r}\right] X_{s}\right)$

(5) $V_{j}\left(\left[Q_{1} / x_{1}, \ldots, Q_{1} / x_{r}\right] X_{1}\right) \ldots\left(\left[Q_{1} / x_{1}, \ldots, Q_{1} / x_{r}\right] X_{s}\right)={ }_{\omega} N$

Observe that $V_{j}\left(\left[V / x_{1}, \ldots, V / x_{r}\right] X_{1}\right) \ldots\left(\left[V / x_{1}, \ldots, V / x_{r}\right] X_{s}\right)$ is still in whnf since this class is closed under internal reductions. This ends the proof of Subcase 2.1.

Subcase 2.2. Otherwise.

Then the endpiece of the desired cascaded proof is as follows:

(1) $M \longrightarrow{ }_{w \beta \Omega}^{*}\left[V / x_{1}, \ldots, V / x_{r}\right] X$

(2) $\left[V / x_{1}, \ldots, V / x_{r}\right] X \quad w \beta \Omega \longleftarrow$

$w \beta \Omega \longleftarrow\left(\lambda x \cdot\left[x / x_{1}, \ldots, x / x_{r}\right] X\right) V$

(3) $\left(\lambda x \cdot\left[x / x_{1}, \ldots, x / x_{r}\right] X\right) V={ }_{\omega}$

$\left(\lambda x .\left[x / x_{1}, \ldots, x / x_{r}\right] X\right) Q_{1}$

(4) $\left(\lambda x .\left[x / x_{1}, \ldots, x / x_{r}\right] X\right) Q_{1} \longrightarrow{ }_{w \beta \Omega}$

$\longrightarrow w \beta \Omega\left[Q_{1} / x_{1}, \ldots, Q_{1} / x_{r}\right] X$ 
(5) $\left[Q_{1} / x_{1}, \ldots, Q_{1} / x_{r}\right] X={ }_{\omega} N$

This ends the proof of Subcase 2.2, and the proof of Proposition 7.5 is complete.

In the following lemma, we recall that $M$ and $N$ (possibly with indexes) always stand for closed terms.

Lemma 7.6. Suppose that:

(1) $U_{1}, U_{2}$ contain the free variable $u$ and no other free variable;

(2) $V_{1}, V_{2}$ contain at most the free variable $u$ and no other free variable;

(3) $V_{1} U_{1} M_{1} \ldots M_{m}$ and $V_{2} U_{2} N_{1} \ldots N_{m}$ are solvable;

(4) $\mathcal{T}$ is a cascaded proof, not ending in the $\omega$-rule, of $\lambda u . V_{1} U_{1} M_{1} \ldots M_{m}={ }_{\omega} \lambda u . V_{2} U_{2} N_{1} \ldots N_{m}$.

Then for each $i$, with $1 \leq i \leq m, M_{i}={ }_{\omega} N_{i}$.

Proof. By induction on $\operatorname{ord}(\mathcal{T})$.

Base case. $\operatorname{ord}(\mathcal{T})=1$. In this case no head $\beta$-redex with a reduct of $U_{i}, i=1,2$, as the argument can be contracted as a weak $\beta$-redex. Neither $U_{i}$ can be part of a head weak $\boldsymbol{\Omega}$-redex. Thus the proof contains weak $\beta \boldsymbol{\Omega}$-conversions of the $M_{i}$ to the $N_{i}$.

Induction step. $\operatorname{ord}(\mathcal{T})$ is infinite. We can freely assume that $\mathcal{T}$ has the form:

$$
\begin{gathered}
\lambda u . V_{1} U_{1} M_{1} \ldots M_{m} \longrightarrow{ }_{w \beta \Omega}^{*} R_{1} \quad w \beta \Omega \longleftarrow L_{1} P_{1}={ }_{\omega} \\
={ }_{\omega} L_{1} Q_{1} \longrightarrow{ }_{w \beta \Omega}^{*} R_{2}{ }_{w \beta \Omega} \longleftarrow{ }_{2} P_{2}={ }_{\omega} L_{2} Q_{2} \longrightarrow_{w \beta \Omega}^{*} \ldots L_{t} P_{t}={ }_{\omega} \\
={ }_{\omega} L_{t} Q_{t} \longrightarrow{ }_{w \beta \Omega}^{*} R_{t+1}{ }_{w \beta \Omega}^{*} \longleftarrow \lambda u \cdot V_{2} U_{2} N_{1} \ldots N_{m} .
\end{gathered}
$$

We claim that each $R_{j}$, with $1 \leq j \leq t+1$, must have the form $\lambda u . V_{1 j}^{\prime} U_{1 j}^{\prime} M_{1 j}^{\prime} \ldots M_{m j}^{\prime}$, for some $V_{1 j}^{\prime}, U_{1 j}^{\prime}, M_{1 j}^{\prime}, \ldots, M_{m j}^{\prime}$, with $\lambda u \cdot V_{1 j}^{\prime}={ }_{\omega} \lambda u . V_{1}, \lambda u \cdot U_{1 j}^{\prime}={ }_{\omega} \lambda u \cdot U_{1}$, and, for $1 \leq i \leq m$, $M_{i j}^{\prime}={ }_{\omega} M_{i}$.

To prove the claim observe that it is true for $R_{1}$. So let $R_{1} \equiv \lambda u . V_{11}^{\prime} U_{11}^{\prime} M_{11}^{\prime} \ldots M_{m 1}^{\prime}$. Consider now $R_{2}$. Since $\mathcal{T}$ is cascaded, the reduction $R_{1} \quad w \beta \Omega \longleftarrow L_{1} P_{1}$ implies that $L_{1}$ has the form $\lambda x u . V_{1}^{*} U_{1}^{*} X_{1}^{*} \ldots X_{m}^{*}$ and that $V_{11}^{\prime} \equiv\left[P_{1} / x\right] V_{1}^{*}, U_{11}^{\prime} \equiv\left[P_{1} / x\right] U_{1}^{*}$, and, for $1 \leq i \leq m$, $M_{i 1}^{\prime} \equiv\left[P_{1} / x\right] X_{i}^{*}$. So, it is clear that $R_{2}$ has the required form. Repeating this argument we get the claim.

By the claim, it follows that $\mathcal{T}$ contains a proof of $M_{i}={ }_{\omega} N_{i}$ for each $i$, with $1 \leq i \leq m$.

\section{Barendregt's Construction}

The present Section requires acquaintance with Section 17.4 of [4. However, we will modify Barendregt's construction in a number of minor points, in order to have a better control of the behavior of the terms. On the other hand, the two constructions are almost identical, and we hope that the reader could be able to reconstruct the correspondences between them.

Assume that an effective coding of finite sequences of natural numbers with natural numbers has been fixed. We call the coding numbers sequence numbers and we denote them by symbols $s, s^{\prime}$, etc. We write $s^{\prime} \leq s\left(s^{\prime}<s\right)$ to denote that $s^{\prime}$ is a subsequence (resp. a proper subsequence) of $s$. Let $f$ be a function from natural numbers to natural numbers; following 
again [4, we denote by $\bar{f}(n)$ the sequence number of the sequence $\langle f(0), \ldots, f(n-1)\rangle$. Now, let $P(n)$ be a $\Pi_{1}^{1}$ predicate. Then:

$$
P(n) \Longleftrightarrow \forall f \exists m R(\bar{f}(m), n)
$$

for some recursive relation $R$. A sequence number $s$ is $n$-secured iff $\exists s^{\prime}<s . R\left(s^{\prime}, n\right)$, otherwise $n$-unsecured. Observe that, for $n$ fixed the set of $n$-unsecured sequence numbers is closed under the subsequence relation and therefore is a tree (possibly empty). Thus $P(n)$ holds iff this tree is well-founded, i.e. not $s_{0}<s_{1}<s_{2} \cdots$ for some infinite sequence of $n$-unsecured sequence numbers. Moreover, the notion " $s$ is $n$-unsecured" is recursive. We can sum up our discussion by the following well known theorem (see [9] Ch.16 Th.20).

Theorem 8.1. The set of (indices of) well founded recursive trees is $\mathbf{\Pi}_{1}^{\mathbf{1}}$-complete.

Now, let again the $\Pi_{1}^{1}$ predicate $P(n)$ be fixed. Let $n$ be fixed once for all, we denote by $T$ the tree of all $n$-unsecured sequence numbers. Now we recall (a version of) Lemma 17.4.11 of [4].

Lemma 8.2. There is a closed term $\square$ such that:

$$
\square \underline{s}={ }_{\omega} \begin{cases}\mathbf{K}^{*} & \text { if } s \in T \\ \Omega & \text { otherwise }\end{cases}
$$

where $\mathbf{K}^{*} \equiv \lambda a b . b$.

Proof. The lemma follows from the fact that $T$ is recursive (see 16.1 .10 of [4]).

As shown by Lemma 17.4.11 of [4], one can have a term $\square$ which is uniform in $n$, i.e. such that, given $n$, it returns a term representing the corresponding tree. More in general, Barendregt shows that all the construction can be done uniformly in $n$. To simplify a little the construction, we have everywhere suppressed this dependency. This will not affect our results.

On the other hand, we need the following slightly stronger version of the previous lemma:

Lemma 8.3. There is a closed term $D$ such that:

(1) For every numeral $\underline{m}, D x \underline{m}$ has a $\beta \boldsymbol{\Omega}$-normal form beginning with $x$ and containing $\underline{m}$. (Where $\underline{m}$ is a parameter needed in the following).

(2) For every $\underline{s}$

$$
D \underline{s}={ }_{\omega} \begin{cases}\mathbf{K}^{*} & \text { if } s \in T \\ \boldsymbol{\Omega} & \text { otherwise }\end{cases}
$$

(3) if s belongs to the tree, then $D \underline{s} \longrightarrow{ }_{w \beta \Omega}^{*} \mathbf{K}^{*}$ by head weak $\beta$-reductions.

Proof. First of all, we can assume that $\square$ of Lemma 8.2 has the property that if $s$ belongs to the tree, then $\square \underline{s} \longrightarrow_{w \beta \Omega}^{*} \mathbf{K}^{*}$ by head weak $\beta$-reductions. This can be obtained by the representation of recursive functions by $\lambda$-terms. We can also assume that $\square$ has the form $\lambda x . X$. Transform the term $X$ as in [12], by replacing (inside out) each $\beta$-redex in $X$ of the form $(\lambda z . Z) W$ into the term $x \mathbf{I I}(\lambda z . Z) W$, where $\mathbf{I}$ is the identity combinator. Let $Y$ be the resulting term, which is obviously in $\beta$-normal form and let $D \equiv \lambda x . x \mathbf{I I} Y$. Observe that for every term $\underline{s}$ (actually a numeral) representing a sequence number we have that:

$$
\underline{s} \mathbf{I I} \longrightarrow{ }_{w \beta \Omega}^{*} \mathbf{I} \text {, by head weak } \beta \text {-reductions, }
$$


so that for every $\underline{s}$ :

$$
D \underline{s} \longrightarrow{ }_{w \beta \Omega}^{*} \mathbf{K}^{*}, \quad \text { by head weak } \beta \text {-reductions, if } s \in T,
$$

and:

$$
D \underline{s} \longrightarrow{ }_{w \beta \Omega}^{*} \Omega, \quad \text { otherwise. }
$$

Finally, it is obvious that for every numeral $\underline{m}, D x \underline{m}$ has a $\beta \boldsymbol{\Omega}$-normal form beginning with $x$ and containing $\underline{m}$.

We now come back to the representation of sequences. For simplicity we denote the term representing the concatenation function by the infixed operator $*$. We can freely require that $\underline{s} * z$ has a $\beta \boldsymbol{\Omega}$ normal form beginning with $z$ (using the same technique of the previous proof).

Now, we define several terms.

(1) $\boldsymbol{\Theta} \equiv(\lambda a b . b(a a b))(\lambda a b . b(a a b))$ (Turing's fixed point).

(2) $Z \equiv \mathbf{\Theta}\left(\lambda a x f .\left[f x, x(\lambda u . u \boldsymbol{\Omega}) y\left(a\left(x^{+}\right) f\right)\right]\right)$, where by $x^{+}$we denote the application of the successor function to $x$. Observe that $y$ is free in $Z$.

(3) $F_{0} \equiv \lambda w x y . D x \underline{0}(\lambda a b . b(a a b))(\lambda a b . b(a a b))\left(\lambda a x f \cdot\left[f x, x(\lambda u . u \boldsymbol{\Omega}) y\left(a\left(x^{+}\right) f\right)\right]\right) \underline{0}(\lambda z . w(x * z))$

(4) $B_{0} \equiv \boldsymbol{\Theta} F_{0}$

(5) $F_{1} \equiv \lambda w x y . D x \underline{1}(\lambda a b . b(a a b))(\lambda a b . b(a a b))\left(\lambda a x f \cdot\left[f x, x(\lambda u . u \boldsymbol{\Omega}) y\left(a\left(x^{+}\right) f\right)\right]\right) \underline{0}(\lambda z . w(x * z))$

(6) $B_{1} \equiv \boldsymbol{\Theta} F_{1}$

First of all observe that both $\Theta$ and $Z$ are not subterms of $F_{0}$ (and neither of $F_{1}$ ); however these terms are generated during the reduction of $F_{0}$ and $F_{1}$ (see below). To relate the previous definitions to [4 page 463, we first observe that $Z$ (with suitable arguments) behaves like the term $\Pi$ of 17.4 .8 of $[4$. Indeed the following lemma holds.

Lemma 8.4. For all $M, N$ and natural number $m$ the following are equivalent:

- for every $P,([P / y] Z) \underline{m} M={ }_{\omega}([P / y] Z) \underline{m} N$

- for every natural number $m^{\prime}$, with $m \leq m^{\prime} M \underline{m^{\prime}}={ }_{\omega} N \underline{m^{\prime}}$.

Proof. Notice that for every $P$ and $M$, and for every $m$ :

$([P / y] Z) \underline{m} M={ }_{\omega}\left[M \underline{m}, P \boldsymbol{\Omega}^{\sim m}\left(([P / y] Z) \underline{m}^{+} M\right)\right]$

(where notations $\sim m$ and $\left[M_{1}, M_{2}, \ldots\right]$ are as in [4 page 25, and, respectively, page 169) then argue as in Theorem 17.4.9 of [4].

Now, we consider the behavior of $B_{0}$ and $B_{1}$, which correspond (with minor modifications) to the terms $B_{0}^{n}$ and, respectively, $B_{1}^{n}$ of 17.4 .13 of [4].

The terms $B_{0}$ and $B_{1}$ have the same behavior and are distinguishable only by the passive parameters $\underline{0}$ and, respectively, $\underline{1}$.

Consider, e.g., $B_{0}$. We have, with 3 head reduction steps:

$$
\begin{aligned}
& B_{0} \longrightarrow{ }_{w \beta \Omega}(\lambda b . b(\boldsymbol{\Theta} b)) F_{0} \\
& \longrightarrow w \beta \Omega F_{0}\left(\boldsymbol{\Theta} F_{0}\right) \\
& \longrightarrow{ }_{w \beta \Omega} \lambda x y . D x \underline{0}(\lambda a b . b(a a b))(\lambda a b . b(a a b))\left(\lambda a x f \cdot\left[f x, x(\lambda u . u \boldsymbol{\Omega}) y\left(a\left(x^{+}\right) f\right)\right]\right) \underline{0}\left(\lambda z \cdot B_{0}(x * z)\right)
\end{aligned}
$$


and if $s$ belongs to the tree then, with a sequence of head weak $\beta$-reductions:

$$
\begin{aligned}
B_{0} \underline{s} & \longrightarrow{ }_{w \beta \Omega}^{*} \lambda y \cdot D \underline{s 0}(\lambda a b \cdot b(a a b))(\lambda a b \cdot b(a a b))\left(\lambda a x f \cdot\left[f x, x(\lambda u \cdot u \boldsymbol{\Omega}) y\left(a\left(x^{+}\right) f\right)\right]\right) \underline{0}\left(\lambda z \cdot B_{0}(\underline{s} * z)\right) \\
& \longrightarrow{ }_{w \beta \Omega}^{*} \lambda y \cdot \boldsymbol{\Theta}\left(\lambda a x f \cdot\left[f x, x(\lambda u \cdot u \Omega) y\left(a\left(x^{+}\right) f\right)\right]\right) \underline{0}\left(\lambda z \cdot B_{0}(\underline{s} * z)\right) \\
& \longrightarrow{ }_{\beta}^{*}(\text { head } \beta \text {-reductions }) \lambda y \cdot\left[\left(\lambda z \cdot B_{0}(\underline{s} * z)\right) \underline{0}, \underline{0}(\lambda u \cdot u \boldsymbol{\Omega})\left(y\left(Z\left(\underline{0}^{+}\right)\left(\lambda z \cdot B_{0}(\underline{s} * z)\right)\right)\right)\right] \\
& \longrightarrow{ }_{w \beta \Omega}^{*} \lambda y \cdot\left[B_{0}(\underline{s} * \underline{0}), y\left(Z\left(\underline{0}^{+}\right)\left(\lambda z \cdot B_{0}(\underline{s} * z)\right)\right)\right]
\end{aligned}
$$

We refer to the reduct $\lambda y \cdot\left[\left(\lambda z \cdot B_{0}(\underline{s} * z)\right) \underline{0}, \underline{0}(\lambda u . u \Omega)\left(y\left(Z\left(\underline{0}^{+}\right)\left(\lambda z \cdot B_{0}(\underline{s} * z)\right)\right)\right)\right]$ as the pivot point, and similarly for $B_{1}$ and $B_{1} \underline{s}$. So, a head reduction of $B_{0} \underline{s}$ or $B_{1} \underline{s}$ begins with 3 head reductions followed by a head reduction of $D \underline{s}$ which either terminates in $\mathbf{K}^{*}$ or fails to terminate. In the first case the next head reduction is of the $\mathbf{K}^{*} \underline{i}(i=0,1)$ redex followed by $\mathbf{I}(\lambda a b . b(a a b)) \longrightarrow w_{w \beta \Omega}(\lambda a b . b(a a b))$ and $\boldsymbol{\Theta} \longrightarrow{ }_{w \beta \Omega} \lambda b . b(\boldsymbol{\Theta} b)$. In the weak $\beta \boldsymbol{\Omega}$ case this is the end of the head reduction sequence since $y$ is contained in the argument of the head redex. In unrestricted $\beta \boldsymbol{\Omega}$-reduction there are 3 more reductions to the pivot point. This ends the description of the behavior of terms $B_{0}$ and $B_{1}$.

Let $T(s)$ denote the subtree of $T$ rooted at the sequence $s$. Here we include the empty tree, in case $s$ is not in $T$. As in [4] 17.4.14, we have that:

Theorem 8.5. For every sequence $s$, if $T(s)$ is well founded then $B_{0} \underline{s}={ }_{\omega} B_{1} \underline{s}$

Proof. Actually in [4] 17.4.14, this is proved for the empty sequence \langle\rangle . However the same proof carries on, since for every sequence $s^{\prime}$, with $s \leq s^{\prime}$ :

- if $s^{\prime} \notin T$ then $B_{0} \underline{s^{\prime}}={ }_{\omega} \boldsymbol{\Omega}={ }_{\omega} B_{1} \underline{s}^{\prime}$;

- if $s^{\prime} \in T$ then, as shown above, $B_{0} \underline{s}^{\prime}={ }_{\omega} \lambda y \cdot\left[B_{0}\left(\underline{s}^{\prime} * \underline{0}\right), y\left(Z\left(\underline{0}^{+}\right)\left(\lambda z \cdot B_{0}\left(\underline{s}^{\prime} * z\right)\right)\right)\right]$ and $B_{1} \underline{s}^{\prime}={ }_{\omega} \lambda y \cdot\left[B_{1}\left(\underline{s^{\prime}} * \underline{0}\right), y\left(Z\left(\underline{0}^{+}\right)\left(\lambda z \cdot B_{1}\left(\underline{s^{\prime}} * z\right)\right)\right)\right]$.

On the other hand, by Lemma 8.4, if for every $m, B_{0}\left(\underline{s^{\prime}} * \underline{m}\right)={ }_{\omega} B_{1}\left(\underline{s^{\prime}} * \underline{m}\right)$ then for every $P,([P / y] Z) \underline{0}\left(\lambda z \cdot B_{0}\left(\underline{s^{\prime}} * z\right)\right)={ }_{\omega}([P / y] Z) \underline{0}\left(\lambda z \cdot B_{1}\left(\underline{s^{\prime}} * z\right)\right)$. By the $\omega$-rule it follows that: $\lambda y \cdot Z \underline{0}\left(\lambda z \cdot B_{0}\left(\underline{s^{\prime}} * z\right)\right)={ }_{\omega} \lambda y \cdot Z \underline{0}\left(\lambda z \cdot B_{1}\left(\underline{s^{\prime}} * z\right)\right)$.

But $\lambda y \cdot \bar{Z} \underline{0}\left(\lambda z \cdot B_{0}\left(\underline{s}^{\prime} * z\right)\right)={ }_{\omega} B_{0} \underline{s^{\prime}}$ and $\lambda y \cdot \bar{Z} \underline{0}\left(\lambda z \cdot B_{1}\left(\underline{s}^{\prime} * z\right)\right)={ }_{\omega} B_{1} \underline{s}^{\prime}$, and thus $B_{0} \underline{s^{\prime}}={ }_{\omega}$ $B_{1} \underline{s}^{\prime}$.

Now argue by bar induction as in [4] 17.4.14.

Theorem 8.6. For every sequence $s$, if $B_{0} \underline{s}={ }_{\omega} B_{1} \underline{s}$ then $T(s)$ is well founded.

Proof. By induction on the ordinal $\operatorname{ord}(\mathcal{T})$ of a cascaded proof $\mathcal{T}$ of $B_{0} \underline{s}={ }_{\omega} B_{1} \underline{s}$. We shall assume that the weak head normal form restrictions on confluence terms are in effect.

Base case. $\operatorname{ord}(\mathcal{T})=1$. Under the hypothesis that $\operatorname{ord}(\mathcal{T})=1$ we have that $B_{0} \underline{s}$ and $B_{1} \underline{s}$ weak $\beta \boldsymbol{\Omega}$-convert. We shall show that $s$ does not belong to $T$ and that $B_{0} \underline{s}=\omega \boldsymbol{\Omega}={ }_{\omega} B_{1} \underline{s}$. We proceed by induction on the lengths of standard $\beta \boldsymbol{\Omega}$-reductions to a common reduct (note here that standardization does not in general hold for weak $\beta \boldsymbol{\Omega}$-reduction so we revert to plain $\beta \boldsymbol{\Omega}$ ). Assume that $s$ actually belongs to $T$. First we show that both reductions must proceed all the way to the pivot point. Clearly both head reductions must complete the head reduction of $D \underline{s}$ and the reduction $\mathbf{K}^{*} \underline{i} \longrightarrow{ }_{w \beta \Omega} \mathbf{I}$ to project the index $\underline{i}$ (where $i=0,1$ ). Since each succeeding term in the head reduction to the pivot point has, respectively, 5, 4, 3, 2, 1 components, if both reductions to the pivot point are not completed then they must stop to a term with the same number of components. It follows that $\lambda z \cdot B_{0}(\underline{s} * z)$ and $\lambda z \cdot B_{1}(\underline{s} * z)$ have shorter standard $\beta \boldsymbol{\Omega}$-reductions to a common reduct. But this is clearly impossible by the conditions on $D$ and $*$, which imply that $\lambda z \cdot B_{0}(\underline{s} * z)$ and $\lambda z \cdot B_{1}(\underline{s} * z)$ 
have Böhm trees which are not $\eta$-equal. Thus both reductions proceed to the pivot point. Thus there are shorter standard confluent $\beta \boldsymbol{\Omega}$-reductions from:

$$
\left(\lambda z \cdot B_{0}(\underline{s} * z)\right) \underline{0} \text { and }\left(\lambda z \cdot B_{1}(\underline{s} * z)\right) \underline{0},
$$

and from:

$$
\underline{0}(\lambda u . u \boldsymbol{\Omega}) y\left(Z\left(\underline{0}^{+}\right)\left(\lambda z \cdot B_{0}(\underline{s} * z)\right)\right) \quad \text { and } \quad \underline{0}(\lambda u . u \boldsymbol{\Omega}) y\left(Z\left(\underline{0}^{+}\right)\left(\lambda z \cdot B_{1}(\underline{s} * z)\right)\right) .
$$

In particular by similar reasoning there exists shorter confluent standard reductions from:

$$
\boldsymbol{\Theta}\left(\lambda a x f .\left[f x, x(\lambda u . u \boldsymbol{\Omega}) y\left(a\left(x^{+}\right) f\right)\right]\right)\left(\underline{0}^{+}\right)\left(\lambda z . B_{0}(\underline{s} * z)\right)
$$

and

$$
\boldsymbol{\Theta}\left(\lambda a x f \cdot\left[f x, x(\lambda u . u \boldsymbol{\Omega}) y\left(a\left(x^{+}\right) f\right)\right]\right)\left(\underline{0}^{+}\right)\left(\lambda z . B_{1}(\underline{s} * z)\right) .
$$

Now we can repeat the above argument with minor modifications forever since no $\Omega$ reductions are possible. This is impossible and proves that $s$ cannot be in $T$.

Induction step. $\operatorname{ord}(\mathcal{T})$ is infinite.

We distinguish twocases.

Case 1. $\mathcal{T}$ ends with a direct conclusion of the $\omega$-rule.

Thus for each closed term $M$ and any sequence $H_{1} \ldots H_{t}$ of closed terms:

$$
B_{0} \underline{s} M H_{1} \ldots H_{t}={ }_{\omega} B_{1} \underline{s} M H_{1} \ldots H_{t}
$$

has a cascaded proof of ordinal smaller than $\operatorname{ord}(\mathcal{T})$. To see this observe that for any $M$, $B_{0} \underline{s} M={ }_{\omega} B_{1} \underline{s} M$ has a cascaded proof of ordinal, say $\gamma$, smaller than $\operatorname{ord}(\mathcal{T})$, which is - by definition of $\operatorname{ord}(\mathcal{T})$ - of the form $\omega^{\theta}$ for some $\theta>\gamma$. Actually, for every $k, \theta>\gamma \oplus k$. Now, $B_{0} \underline{s} M H_{1} \ldots H_{t}={ }_{\omega} B_{1} \underline{s} M H_{1} \ldots H_{t}$ can be obtained from the endpiece:

$B_{0} \underline{s} M H_{1} \ldots H_{t} \sim_{w \beta \Omega}\left(\lambda x . x H_{1} \ldots H_{t}\right)\left(B_{0} \underline{s} M\right)=_{\omega}\left(\lambda x . x H_{1} \ldots H_{t}\right)\left(B_{1} \underline{s} M\right) \sim_{w \beta \Omega} B_{1} \underline{s} M H_{1} \ldots H_{t}$, which clearly has a cascaded proof of ordinal smaller than $\gamma \oplus k$, for some $k$, and therefore smaller than $\omega^{\theta}$.

Now for any $m$, we can choose $M, H_{1}, \ldots H_{t_{m}}$ to Böhm out, as in Theorem 17.4.9 of [4], $B_{0}(\underline{s} * \underline{m})$ and $B_{1}(\underline{s} * \underline{m})$ from $B_{0} \underline{s}$ and, respectively, $B_{1} \underline{s}$.

Thus for each $m, B_{0}(\underline{s} * \underline{m})={ }_{\omega} B_{1}(\underline{s} * \underline{m})$ is provable by a proof with ordinal smaller than $\operatorname{ord}(\mathcal{T})$. Hence, by induction hypothesis, the subtree $T(s * m)$ of $T$ rooted at $s * m$ is well founded. It follows that the subtree of $T$ rooted at $s$ is well founded as well.

Case 2. Otherwise.

So $\mathcal{T}$ has an endpiece. Since $\mathcal{T}$ is cascaded, the endpiece has the form:

$$
\begin{gathered}
B_{0} \underline{s} \longrightarrow{ }_{w \beta \Omega}^{*} R_{1} \quad{ }_{w \beta \Omega} \longleftarrow M_{1} P_{1}={ }_{\omega} M_{1} Q_{1} \longrightarrow{ }_{w \beta \Omega}^{*} R_{2} \quad w \beta \Omega \longleftarrow M_{2} P_{2}={ }_{\omega} M_{2} Q_{2} \longrightarrow{ }_{w \beta \Omega}^{*} \\
\ldots{ }_{w \beta \Omega}^{*} R_{t+1}{ }_{w \beta \Omega}^{*} \longleftarrow B_{1} \underline{s} .
\end{gathered}
$$

Where each $R_{i}$ is in whnf and all left-arrow reductions (with the possible exception of the last one) are one step weak $\beta$-reductions not of the form $\left(\lambda x y_{1} \ldots y_{r} . x X_{1} \cdots X_{m}\right) U$.

We shall show that also in this case $s$ is not in $T$. By contradiction, assume $s$ in $T$. Since the weak head normal form restrictions are in effect, the head reduction part of the reduction to $R_{1}$ terminates in:

$$
\lambda y . \lambda b . b(\mathbf{\Theta} b)\left(\lambda a x f \cdot\left[f x, x(\lambda u . u \boldsymbol{\Omega}) y\left(a f\left(x^{+}\right)\right)\right]\right) \underline{0}\left(\lambda z . B_{0}(\underline{s} * z)\right)
$$

and similarly the head reduction part of the reduction from $B_{1} \underline{s}$ to $R_{t+1}$ terminates in:

$$
\lambda y . \lambda b . b(\boldsymbol{\Theta} b)\left(\lambda a x f .\left[f x, x(\lambda u . u \boldsymbol{\Omega}) y\left(a f\left(x^{+}\right)\right)\right]\right) \underline{0}\left(\lambda z . B_{1}(\underline{s} * z)\right) .
$$


Now, let:

- $V_{1} \equiv V_{2} \equiv \lambda b . b(\boldsymbol{\Theta} b)$

- $U_{1} \equiv U_{2} \equiv \lambda a x f .\left[f x, x(\lambda u . u \boldsymbol{\Omega}) y\left(a f\left(x^{+}\right)\right)\right]$

- $M_{1} \equiv N_{1} \equiv \underline{0}$

- $M_{2} \equiv \lambda z \cdot B_{0}(\underline{s} * z)$

- $N_{2} \equiv \lambda z \cdot B_{1}(\underline{s} * z)$

Thus by the Lemma 7.6 there exists a proof of $\lambda z \cdot B_{0}(\underline{s} * z)={ }_{\omega} \lambda z \cdot B_{1}(\underline{s} * z)$. But this is impossible because these terms have Böhm trees which are not $\eta$-equal. This completes the proof.

Corollary 8.7. The set $\left\{(M, N) \mid M={ }_{\omega} N\right\}$ is $\boldsymbol{\Pi}_{1}^{1}$-complete.

Proof. Let $P(n)$ be a $\Pi_{1}^{1}$ predicate. Given any natural number $n$, to compute the truth value of $P(n)$, construct the recursive tree $T$ of all $n$-unsecured sequence numbers. Then construct the terms $B_{0}$ and $B_{1}$. Then use Theorem 8.5 and Theorem 8.6 to determine (via equality in $\mathcal{H}(\omega)$ if $T$ is well founded.

\section{ACKNOWLEDGEMENT}

We thank the anonymous referee for her/his help in substantially improving a previous version of the paper.

\section{REFERENCES}

[1] URL: http://www.cl.cam.ac.uk/Research/HVG/HOL.

[2] URL: http://www.cl.cam.ac.uk/Research/HVG/Isabelle.

[3] URL: http://coq.inria.fr.

[4] H.P. Barendregt. The Lambda Calculus. Its Syntax and Semantics. North-Holland, 1984.

[5] R.C. Flagg, J. Myhill. Implication and Analysis in Classical Frege Structure. Annals of Pure and Applied Logic 34 (1987) pp.33-85.

[6] B. Intrigila, R. Statman. Some Results on Extensionality in Lambda Calculus. Annals of Pure and Applied Logic. 132, Issues 2-3, (2005) pp.109-125.

[7] B. Intrigila, R. Statman. The Omega Rule is $\Pi_{2}^{0}$-Hard in the $\lambda \beta$-Calculus. LICS 2004 pp.202-210, IEEE Computer Society 2004.

[8] B. Intrigila, R. Statman. $\lambda \beta \omega$ is not arithmetical. Unpublished Paper.

[9] H. Rogers, Jr. Theory of Recursive Functions and Effective Computability. MacGraw Hill New York 1967.

[10] K. Schütte Proof Theory Springer Verlag New York Heidelberg Berlin 1977.

[11] R. Statman. Gentzen's notion of a direct proof. in Handbook of Mathematical Logic (K.J. Barwise Editor) North Holland Amsterdam 1978.

[12] R. Statman Normal varieties of combinators. CMU Math. Dept. Research Report No. 88-32. Carnegie-Mellon University 1988.

This work is licensed under the Creative Commons Attribution-NoDerivs License. To view a copy of this license, visit http://creativecommons.org/licenses/by-nd/2.0/ or send a letter to Creative Commons, 559 Nathan Abbott Way, Stanford, California 94305, USA. 